\title{
Undulating periodization models for strength training \& conditioning
}

\section{A. Jiménez}

Physical Activity \& Sports Sciences School, European University of Madrid, Spain

\begin{abstract}
Periodized strength training refers to varying the training program at regular time intervals in an attempt to bring about optimal resistance gains. The main aim of this paper was to present a short review of the diferent aspects of training periodization and its effects with performance. as well as the differential effect of alternate periodization models on other populations, and specially the need for further research regarding the effectiveness of the undulating model as compared with the linear model. A focussed literature review reveals that most studies that examined strength training periodization utilized young males as their subject population, and the research has mainly focused on differences between periodized and non-periodized programs. Furthermore, the periodization training programs are designed and developed according to two different models: the linear model and the non-linear model. The Linear Model is characterized by high initial training volume and low and intensity. The Non-Linear Model enables variation in intensity and volume within each 7-10 day cycle by rotating different protocols to train various components of the neuromuscular system. The results showed at the scientific literature encourage researchers and exercise professionals to include non-linear (undulating) periodization models during resistance training.
\end{abstract}

Key words: periodization, resistance, training, power

Periodized strength training refers to varying the training program at regular time intervals in an attempt to bring about optimal gains in strength, power, motor performance, and/or muscle hypertrophy (Fleck, 1999). To date, all studies published indicate that it does result in significant fitness gains and results in greater gains than other training models provide (Kraemer \& Fleck, 2007). Despite the many different versions of periodization that the previous researchers and coaches have used in the literature, periodization is a concept that can be defined by programmed variation in the training stimuli with the use of planned rest periods to augment recovery and restoration of an athlete's potential. In fact, periodization, representing planned variations of the training variables (i.e., volume, intensity, frecuency, etc.), is one of the most written and talked about topics in strength and conditioning circles (Rhea \& Alderman, 2004).

It has been shown that greater volumes and training intensities result in strength greater adaptations, up to a certain level. The mechanisms and reasoning behind improvements in strength and power adaptations due solely to training variation are somewhat unclear. These strength increases are presumably due to greater overload of the neuromuscular system (Rhea, Ball, Philips \& Burkett, 2002). Based on this line of reasoning, independent of 
increases in training volume and intensity, may increase the overload the neuromuscular system experiences by continually applying an un-customized stress.

Periodized resistance training has demonstrated effectiveness on sports performance as well as recreation training (Dolezal \& Potteiger, 1998) and rehabilitation (Fees, Decker \& Snyder-Mackler, 1998). In a meta-analysis review published by Rhea and Alderman (2004), the Periodized programs, consider as a whole (i.e., including the ability to train at higher volumes and intensities), elicits a greater increase in strength and power that nonperiodized training (Effect Size, ES $=0.84$ ). Moreover, another analysis by the same authors demonstrated that, when volume and intensity are similar, periodized training still elicits greater improvements than non-periodized training (ESs $=1.28$ and 1.03 , respectively) (Rhea \& Alderman, 2004). Despite the use of periodized strength training by the athletic community for at least 40 years, few published sport science projects and studies have investigated the efficacy of periodized strength training (Fleck, 1999).

A focussed literature review reveals that most studies that examined strength training periodization utilized young males as their subject population, and the research has mainly focused on differences between periodized and non-periodized programs. Therefore, it is necessary to investigate varying aspects of periodized training as well as the differential effect of alternate periodization models on other populations, and specially the need for further research regarding the effectiveness of the undulating model as compared with the linear model (Fleck, 1999; Rhea et al., 2002; Rhea \& Alderman, 2004; Tan, 1999). Fleck (1999) contended that the additional strength gains elicited by Periodization training could be related to greater training volumes when following such training models (Rhea \& Alderman, 2004). Usually, the Periodization training programs are designed and developed according to two different models: the linear model and the non-linear model.

The Linear Model is characterized by high initial training volume and low and intensity. As training progresses, volume decreases and intensity increases in order to maximize strength, power, or both (Fleck, 1999).

The Undulating (Non-Linear) Model enables variation in intensity and volume within each 710 day cycle by rotating different protocols to train various components of the neuromuscular system (e.g. strength, power, local muscular endurance).

For example, in loading schemes for the core exercises in the workout, the use of heavy, moderate and lighter resistances may be randomly rotated over a training sequence (e.g.: M: 3-5 RM loads; W: 8-10 RM loads; F: 12-15 RM loads). This model has compared favourably with classical periodized and non-periodized multiple set models (Baker, Wilson \& Carlyon, 1994), was shown to produce greater strength increases over 12 weeks of training compared to the classical model (Rhea et al., 2002), and was shown to have distinct advantages in comparison to non-periodized, low volume training in women (Marx et al., 2001). 
For O'Bryant, Byrd and Stone (1998) some have described linear periodization as variation of the training intensity over several weeks of training with nonlinear periodization as variation of the training intensity and volume over a week with differences from day to day. However, by definition, any periodization should be considered nonlinear. Although the general loading process (with respect to initial training levels) overtime may be considered linear, variations in volume and intensity that occur within a microcycle - also represented by microcycles in a mesocycle and mesocycles in a macrocycle - exhibit a nonlinear pattern.

In studies using similar males subjects and training programs, Rhea et al. (2002) reported improvements about $28.78 \%$ in undulating group and $14.37 \%$ in linear group, while Baker et al. (1994) obtained $16.4 \%$ in undulating group and $11.6 \%$ in linear group. These results agree with the obtained in the meta-analysis developed by Rhea and Alderman (2004), where the effectiveness of periodization in strength gains was effective in both sexes, (women show a size effect of 0.99 , very similar to the results obtained in men, 1.02).

Poliquin (1988) theorized that more frecuent changes in stimulus would enhance strength gains. In his original undulated program, alterations were to be made every 2 weeks. Such a program was found to elicit similar strength gains as a linear model program.

From our experience, like at the Rhea's et al. (2002) study, when training variables are altered on a daily basis, as hypothesized, the undulating models seems to demonstrate significantly more strength gains than linear models. For Rhea at al. (2002) these strength increases are presumably due to greater overload of the neuromuscular system, applying an uncustomized stress, which may result in greater fitness gains. In any case, further research is needed on this topic, because the driving mechanisms behind the increased effectiveness of undulating models are not completely understood.

For Kraemer (1997), different from the linear programs is the practice that one trains the different components of muscle size and strength within the same week. Different from the linear methods, nonlinear programs attempt to train different adaptation aspects of the neuromuscular system within the same microcycle. Thus, one is working at different physiological adaptations together within the same 7-to 14-day period of a usual 16-week mesocycle. A rest or restoration cycle then follows this training cycle. This appears possible and may be more conductive to many individuals' schedules, especially when competitions, travel or other schedule conflicts, practice demands, sickness, etc, can make the traditional linear method structures difficult to adhere to and train large groups of athletes.

\section{Conclusions \& practical applications}

The results showed at the scientific literature encourage researchers and exercise professionals to include undulating periodization models during Resistance Training and Conditioning Programs.

The use of this type of training method can be made from two practical perspectives: Foremost is the ability to deal with schedule demands of athletes as well as fitness 
enthusiasts; the secondary concern is readiness to train optimally in a workout (Kraemer \& Fleck, 2007). In many cases the undulating approach provides greater versatility in the development of various mesocycles.

From the actual evidences, it is important to take care about the implementation of undulating periodization models, after an initial adaptation phase. For Kraemer and Fleck (2007), the potential weakness of the undulating program is that heavy loads are implemented in the first week of workout. Thus, the beginner needs to perform a base program for 4 to 6 weeks using lighter weights, allowing the individual to gain toleration to the resistance training program. Then one starts an undulating progression of varying intensities. An active rest or recovery period (to get away from resistance training and remain active with other sporting activities) of 2 to 3 weeks in length could be placed after the 16 -week cycle.

Finally, the most common undulating periodization model sample protocol could be recognized from the proposed by Kraemer and Fleck (2007):

Table 1. Sample protocol of Undulating Periodization in a 16-week mesocycle (Kraemer \& Fleck, 2007)

The protocol uses a 6 -day rotation

\section{Monday}

4 sets of 12 to 15 RM

Wednesday

4 sets of 8 to $10 R M$

Friday

3 or 4 sets of 4 to $6 \mathrm{RM}$

Active rest

For 2 to 3 weeks after the 16 -week

mesocycle is completed

\section{References}

Baker, D., Wilson, G., \& Carlyon, R. (1994). Periodization: The effect on strength of manipulating volume and intensity. Journal of Strength and Conditioning Research, 8 , 235-242.

Dolezal, B.A., \& Potteiger J.A. (1998). Concurrent resistance and endurance training influence basal metabolic rate (BMR) in non-dieting individuals. Journal of Applied Physiology, 85, 695-700.

Fees, M., Decker, L., \& Snyder-Mackler, M.J. (1998). Upper extremity weight-training modifications for the injures athlete: a clinical perspective. American Journal of Sports Medicine, 26, 732-742.

Fleck, S.J. (1999). Periodized strength training: A critical review. Journal of Strength and Condition Research, 13, 82-89. 
Kraemer, W.J. (1997). A series of studies - The physiological basis for strength training in American football: Fact over philosophy. Journal of Strength and Conditioning Research, $11,131-142$.

Kraemer, W.J., \& Fleck, S. (2007). Optimizing strength training. Champaign, IL: Human Kinetics.

Marx, J.O., Ratamess, N.A., Nindl, B.C, Gotshalk, L.A., Volek, J.S., Dohi, K., et al. (2001). The effects of single-set vs. periodized multiple-set resistance training on muscular performance and hormonal concentrations in women. Medicine and Science in Sports and Exercise, 33, 635-643.

O'Bryant, H.S., Byrd, R., \& Stone, M.H. (1988). Cycle ergometer performance and maximum leg and hip strength adaptations to two different methods of weight-training. Journal of Applied Sport Science Research, 2, 27-30.

Poliquin, C. (1988). Five ways to increase the effectiveness of your strength training program. National Strength and Conditioning Association Journal, 10(3), 34-39.

Rhea, M., \& Alderman, B. (2004). A meta-analysis of periodized versus nonperiodized strength and power training programs. Research Quaterly for Exercise and Sports, 75, 413-422.

Rhea, M.R., Ball, S.D., Phillips, W.T., \& Burkett, L.N. (2002). A comparison of linear and daily undulating periodized programs with equated volume and intensity for strength. Journal of Strength and Conditioning Research, 16(2), 250-255.

Tan, B. (1999). Manipulating resistance training program variables to optimize maximum strength in men: A review. Journal of Strength and Conditioning Research, 13, 289-304. 